\title{
Comparison of the effects of minimally invasive percutaneous pedicle screws osteosynthesis and open surgery on repairing the pain, inflammation and recovery of thoracolumbar vertebra fracture
}

\author{
YAHUI GONG, GUANGMIN FU, BO LI, YINAN LI and XIAONING YANG \\ Department of Orthopedics, The First People's Hospital Xuzhou, The Affiliated Hospital \\ of Xuzhou Medical University, Xuzhou, Jiangsu 221000, P.R. China
}

Received April 4, 2017; Accepted August 21, 2017

DOI: $10.3892 /$ etm.2017.5036

\begin{abstract}
We compared the clinical effects of minimally invasive percutaneous pedicle screws osteosynthesis (MIPPSO) and open surgery on the repair of thoracolumbar vertebra fracture. Seventy patients, who suffered from thoracolumbar vertebra fracture and received treatment at our hospital, were selected and randomly divided into either the minimally invasive percutaneous pedicle screws osteosynthesis group (MIPPSO group) and the traditional open pedicle screws osteosynthesis group (TOPSO group) with 35 cases in each group. The perioperative parameters including length of incision, duration of operation, bleeding during operation, length of hospital stay, the changes of pre-operative and postoperative VAS pain scores, inflammatory indexes including serum $\mathrm{C}$-reactive protein $(\mathrm{CRP})$ and creatine kinase $(\mathrm{CK})$, and imaging indexes including Cobb's angle and anterior margin height of vertebral body of both groups were compared. The length of incision, duration of operation, bleeding volume during operation and length of hospital stay in the MIPPSO group were significantly lower than those in the TOPSO group $(\mathrm{P}<0.05)$. Both minimally invasive surgery and traditional surgery effectively alleviated the pain $(\mathrm{P}<0.05)$, which was more significant in MIPPSO group $(\mathrm{P}<0.05)$. The postoperative inflammatory indexes, CRP and CK levels, of both groups were higher compared to the pre-operation $(\mathrm{P}<0.05)$, which was more significant in TOPSO group $(\mathrm{P}<0.05)$. The differences of imaging indexes, including Cobb's angle and anterior margin height of injured vertebra, were statistically significant between pre-operation and post-operation for each
\end{abstract}

Correspondence to: Dr Xiaoning Yang, Department of Orthopedics, The First People's Hospital Xuzhou, The Affiliated Hospital of Xuzhou Medical University, 19 Zhongshan North Road, Xuzhou, Jiangsu 221000, P.R. China

E-mail: yang2doc@163.com

Key words: minimally invasive percutaneous pedicle screws osteosynthesis, Cobb's angle, anterior margin height of vertebral body, thoracolumbar vertebra fracture group $(\mathrm{P}<0.05)$; however, there were no statistically significant differences between two groups at either pre-operation or post-operation $(\mathrm{P}>0.05)$. The effect of minimally invasive percutaneous pedicle screws osteosynthesis is similar to the traditional open surgery, however, the MIPPSO technique has the advantages of small trauma, less bleeding, short duration of operation, rapid post-operative recovery, light pain, less economic cost, and better aesthetic effect and is therefore worthy of clinical promotion.

\section{Introduction}

With the rapid development of industrialization and an increasing number of traffic accidents, the incidence rate of spinal fractures is constantly rising, reaching as high as 5-6\% of full-body fractures, of which the thoracolumbar fracture is the most common. Thoracolumbar vertebral fracture refers to the state that bone cortical continuity is impaired by an external force (1), which is manifested by local pain. Without prompt treatment, nerve damage (motion and sense dysfunctions of double lower limbs, excretion dysfunction) may occur, thereby causing more serious complications (2). The traditional open osteosynthesis shows increased trauma, massive bleeding, long length of hospital stay and multiple complications (3). Moreover, the ischemic necrosis and fibrosis of muscles caused by extensive detachment and repeated sustained traction of tissues adjacent to the vertebral body (fascia muscularis, muscle and anadesma) can seriously affect the prognosis of patients, which may lead to the intractable stiffness of the waist and back. As early as the 1980s, through using percutaneous pedicle screws osteosynthesis, Magerl (4) created the minimally invasive technique for the treatment of spinal trauma. The clinical application of minimally invasive percutaneous pedicle screws osteosynthesis has gradually been popularized in recent years (5). In this study, 70 patients who suffered from spinal fractures and received treatment at our hospital from January 2014 to June 2016 were selected. The minimally invasive percutaneous pedicle screws osteosynthesis and traditional open surgery were used for treatment, which achieved satisfactory clinical effects. 
Table I. Comparison of general conditions of two groups of patients.

\begin{tabular}{lccr}
\hline Item & MIPPSO group & TOPSO group & P-value \\
\hline Sex (Male/Female, $\mathrm{n})$ & $23 / 12$ & $24 / 11$ & $>0.05$ \\
Age (mean $\pm \mathrm{SD}$, years) & $36.89 \pm 7.21$ & $37.01 \pm 6.83$ & $>0.05$ \\
Weight (mean $\pm \mathrm{SD}, \mathrm{kg})$ & $63.26 \pm 9.27$ & $64.54 \pm 8.65$ & $>0.05$ \\
Denis typing (n) & & & $>0.05$ \\
Simple compression fracture & 8 & 9 & $>0.05$ \\
Blowout fracture & 27 & 26 & \\
Injured segment (n) & & 1 & \\
T11 & 2 & 6 & \\
T12 & 7 & 21 & \\
L1 & 20 & 7 & \\
L2 & 6 & & \\
\hline
\end{tabular}

The general conditions of sex distribution, age, weight, Denis typing and injured segment of two groups of patients were statistically analyzed, showing no significant differences $(\mathrm{P}>0.05)$. Baseline characteristics were comparable between two groups.

\section{Materials and methods}

Sample selection. Seventy patients, who suffered from thoracolumbar vertebra fracture with no nerve damage and received treatment at our hospital from January 2014 to June 2016 , were selected and randomly divided into either the minimally invasive percutaneous pedicle screws osteosynthesis group (MIPPSO group) or the traditional open pedicle screws osteosynthesis group (TOPSO group) with 35 cases in each group. The selected patients or their families signed written informed consent form, and the study was approved by the Ethics Committee at Xuzhou Hospital.

The inclusion criteria were as follows: a) the patient was diagnosed with a thoracolumbar vertebra fracture, $b$ ) the patient suffered from Type-A, Grade-E single segmental fracture, with no need for spinal canal decompression, and c) the patient who suffered from fresh fracture within two weeks. The exclusion criteria were as follows: a) the patient with thoracolumbar vertebra fracture who needed spinal canal decompression to relieve nervous symptoms; and b) pathological and/or old fracture (Table I).

Materials. The percutaneous pedicle screw system, which was purchased from Changzhou Kanghui Medical Innovation Co., Ltd. (Changzhou, China), has good biocompatibility, no obvious allergic reaction and no effect on magnetic resonance examination.

Methods. Patients in the MIPPSO group were treated as follows. The patient assumed the position of prone hyperextension, thereby suspending the abdomen. The injured vertebra and the projection of upper and lower vertebral pedicle center on the body surface was orientated and marked by using a C-arm X-ray machine (Siemens, Frankfurt, Germany). The towels were routinely sterilized, and then a total of four longitudinal incisions, $2.0 \mathrm{~cm}$ in length, were made along with the orientation under general anesthesia with endotracheal intubation.
The tissue was cut open layer by layer via a blunt separation to expose the operative field. According to the perspective of the $\mathrm{C}$-arm machine, the screw was kept in parallel with the end plate, keeping a $10-15^{\circ}$ angle of sagittal orientation towards the inside; then, the screw was placed into vertebral pedicle. It was ensured that the inner cortex was not broken through the screw and the position was placed well under the perspective of the $\mathrm{C}$-arm machine. The same method was adopted to place other vertebral pedicle screws. Then, the fixation rods were installed. The pre-curved fixation rods were successively placed into the upper and lower pedicle screw tail grooves via a subcutaneous muscular tunnel. The injured vertebra was reset supporting by the distracter. After confirming the satisfactory reduction, all the nuts were tightened. With no placement of a drainage strip, the incision was sutured layer by layer. In the TOPSO group, surgery was conducted in accordance with the traditional method.

Main observation indexes. The main observation indexes were outlined as follows:

Pre-operative parameters. The pre-operative parameters include the length of surgical incision, duration of operation, bleeding during operation and length of hospital stay of patients in both groups.

Pain assessment. Pain assessment was conducted using the visual analog scale (VAS) scoring system (6). The corresponding point of pain intensity, which the patient felt on the $10 \mathrm{~cm}$ line, was confirmed. The VAS scores of patients in both groups were compared before the operation and on the postoperative 1st, 3rd and 5th day, respectively.

Inflammation-related indexes. Through the respective detection of CRP levels and CK activities in peripheral blood before operation and at post-operative 24 and $48 \mathrm{~h}$, the changes in levels of serum CRP levels and CK activities of 


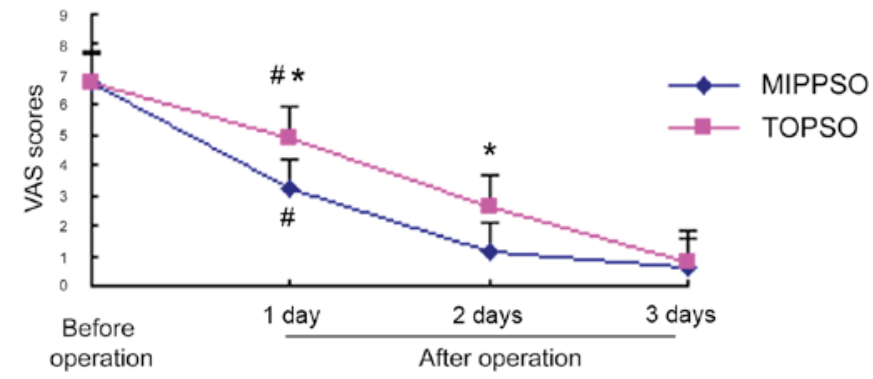

Figure 1. Comparisons of pre-operative and post-operative VAS pain scores of two groups of patients. Compared to the pre-operation, ${ }^{\#} \mathrm{P}<0.05$; compared to the TOPSO group, ${ }^{*} \mathrm{P}<0.05$.

Table II. Comparisons of perioperative parameters of two groups of patients (mean $\pm \mathrm{SD}, \mathrm{n}=35$ ).

\begin{tabular}{|c|c|c|c|}
\hline Item & $\begin{array}{l}\text { MIPPSO } \\
\text { group }\end{array}$ & $\begin{array}{l}\text { TOPSO } \\
\text { group }\end{array}$ & $\mathrm{P}$-value \\
\hline Length of incision $(\mathrm{cm})$ & $2.04 \pm 1.32$ & $9.57 \pm 1.02$ & $<0.05$ \\
\hline Duration of operation (min) & $59.46 \pm 7.28$ & $123.16 \pm 4.65$ & $<0.05$ \\
\hline $\begin{array}{l}\text { Bleeding volume of } \\
\text { operation }(\mathrm{ml})\end{array}$ & $21.31 \pm 9.76$ & $162.35 \pm 30.64$ & $<0.05$ \\
\hline Length of hospital stay (days) & $5.28 \pm 3.50$ & $10.37 \pm 2.33$ & $<0.05$ \\
\hline
\end{tabular}

There was a statistically significant difference in each parameter between the groups, $\mathrm{P}<0.05$.

pre-operation and post-operation of patients in both groups were compared.

Imaging indexes. Through the pre-operative X-ray examination and conducting re-examinations on the post-operative 3rd day and in the 1st year, the changes of Cobb's angles of pre-operation and post-operation of patients in both groups were compared. The anterior margin height of injured vertebra $=$ Actual height $/$ Reference height $\times 100 \%$. Reference height $=$ the sum of upper and lower anterior margin height of injured vertebra $/ 2 \times 100 \%$. The measure of Cobb's angle and sagittal index (SI) referred to the literature (7).

Statistical analysis. The SPSS 18.0 software (SPSS Inc., Chicago, IL, USA) was used for statistical analysis. All data were expressed as mean $\pm \mathrm{SD}$. The t-test and Chi-square test were used to compare the data. $\mathrm{P}<0.05$ was considered to indicate a statistically significant difference.

\section{Results}

Comparisons of perioperative parameters of two groups of patients. The length of surgical incision, duration of operation, bleeding volume of operation and length of hospital stay of patients in both groups were compared. The MIPPSO group had the advantages of small incision, short duration of operation, less bleeding and short hospital stay, and there was a statistically significant difference in each parameter between the groups of data $(\mathrm{P}<0.05)$ (Table II).

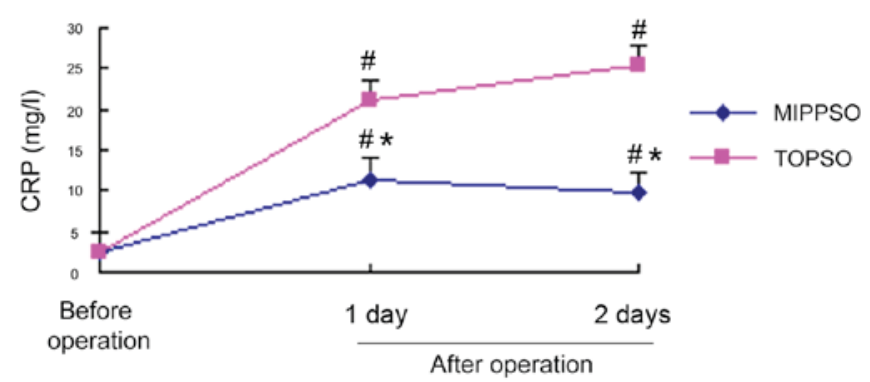

Figure 2. Comparisons of pre-operative and post-operative serum CRP levels of two groups of patients. Compared to the pre-operation, ${ }^{\#} \mathrm{P}<0.05$; compared to the TOPSO group, ${ }^{*} \mathrm{P}<0.05$.

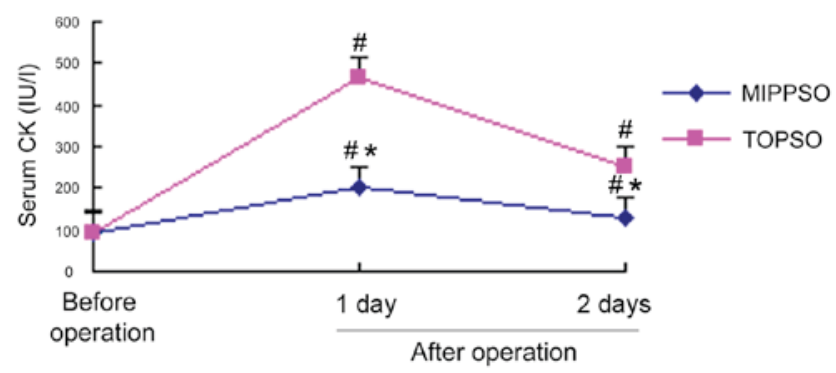

Figure 3. Comparisons of pre-operative and post-operative serum CK levels of two groups of patients. Compared to the pre-operation, ${ }^{\#} \mathrm{P}<0.05$; compared to the TOPSO group, ${ }^{*} \mathrm{P}<0.05$.

Comparisons of VAS pain scores of the groups of patients. The pre-operative VAS pain scores of patients in the MIPPSO group and the TOPSO group were $6.76 \pm 1.80$ and $6.69 \pm 2.01$, respectively, which showed no statistically significant difference $(P>0.05)$. The scores on the post-operative 1 st and 3rd day in the MIPPSO group were, respectively 3.23 \pm 1.50 and $1.14 \pm 1.37$, while the scores on the post-operative 1 st and 3rd day in the TOPSO group were $4.91 \pm 1.52$ and $2.66 \pm 1.14$, respectively. The pain of both groups on the post-operative 1 st and 3rd day were relieved when compared to levels preoperation, with a statistically significant difference $(\mathrm{P}<0.05)$. Compared to the TOPSO group, the ease of pain in the MIPPSO group was more obvious, and the difference was statistically significant $(\mathrm{P}<0.05)$. The pain scores of both groups on the post-operative 5 th day were $0.58 \pm 0.54$ and $0.81 \pm 1.06$, and there was no statistically significant difference (P>0.05) (Fig. 1).

Changes of pre-operative and post-operative inflammatory indexes (serum CRP and CK) of both groups of patients Comparison of changes of pre-operative and post-operative serum CRP of both groups of patients. The baseline serum CRPs of patients in both groups before the operation were comparable. The serum CRPs of patients in the MIPPSO group and the TOPSO group were $11.43 \pm 5.01 \mathrm{mg} / \mathrm{l}$ and $21.16 \pm 5.72 \mathrm{mg} / \mathrm{l}$ at post-operative $24 \mathrm{~h}$, respectively. The concentrations in the MIPPSO group and the TOPSO group were respectively $9.67 \pm 6.25 \mathrm{mg} / \mathrm{l}$ and $25.39 \pm 2.86 \mathrm{mg} / \mathrm{l}$ at post-operative $48 \mathrm{~h}$. The serum CRPs of both groups at 24 and $48 \mathrm{~h}$ after operation were significantly increased when compared to the pre-operation levels $(\mathrm{P}<0.05)$. Moreover, the serum CRPs in the TOPSO 


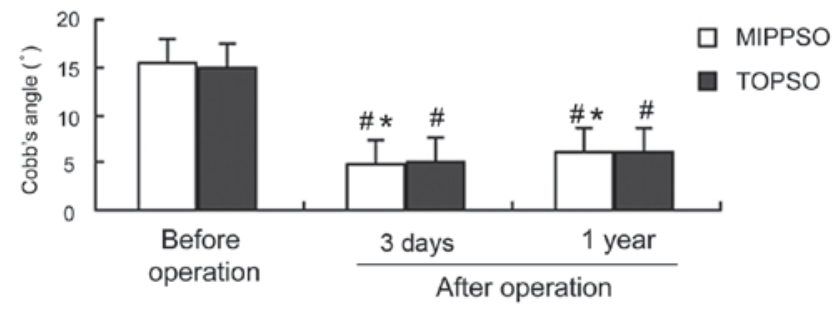

Figure 4. Comparisons of Cobb's angles (imaging index) of two groups of patients. Compared to the pre-operation, ${ }^{\#} \mathrm{P}<0.05$; compared to the TOPSO group, ${ }^{*} \mathrm{P}>0.05$.

group at post-operative 24 and $48 \mathrm{~h}$ were significantly higher than those in the MIPPSO group $(\mathrm{P}<0.05)$ (Fig. 2).

Comparisons of the changes of pre-operative and postoperative serum $C K$ of both groups of patients. The baseline serum CKs of patients in both groups before the operation were comparable. The serum CKs of patients in the MIPPSO group and TOPSO group were 201.34 445.96 IU/1 and $465.61 \pm 45.27 \mathrm{IU} / 1$ at post-operative $24 \mathrm{~h}$, respectively. The concentrations were $129.67 \pm 56.25 \mathrm{IU} / 1$ and $252.39 \pm 42.65 \mathrm{IU} / 1$ at post-operative $48 \mathrm{~h}$, respectively. The serum CKs of both groups at $24 \mathrm{~h}$ post-operation were all significantly increased when compared to the pre-operation $(\mathrm{P}<0.05)$. The concentrations were decreased at post-operative $48 \mathrm{~h}$ but still higher than the pre-operation $(\mathrm{P}<0.05)$. The serum CKs in the TOPSO group were significantly higher than those in the MIPPSO group at post-operative 24 and $48 \mathrm{~h}$ $(\mathrm{P}<0.05)$ (Fig. 3).

Changes of pre-operative and post-operative imaging indexes (Cobb's angle and anterior margin height of vertebral body) of both groups of patients

Comparisons of the changes of pre-operative and postoperative Cobb's angle of two groups of patients. The Cobb's angles of patients in both groups before the operation were $15.5 \pm 5.3^{\circ}$ and $14.9 \pm 5.6^{\circ}$, respectively, which were comparable ( $\mathrm{P}>0.05)$. The Cobb's angles in the MIPPSO group and the TOPSO group were $4.8 \pm 3.5^{\circ}$ and $5.0 \pm 3.3^{\circ}$ on the post-operative $3 \mathrm{rd}$ day, and $6.1 \pm 2.9^{\circ}$ and $6.2 \pm 2.7^{\circ}$ in the post-operative 1st year, respectively. The Cobb's angles of patients in both groups on the 3rd day and in the 1st year post-operation were all significantly decreased when compared to pre-operation $(\mathrm{P}<0.05)$, but there were no statistically significant differences between the groups ( $\mathrm{P}>0.05)$ (Fig. 4).

Comparison of changes of the pre-operative and post-operative anterior margin height of the vertebral body of both groups of patients. The anterior margin heights of the vertebral body of patients in both groups before the operation were $64.3 \pm 11.2 \%$ and $65.9 \pm 9.5 \%$, respectively, which were comparable $(\mathrm{P}>0.05)$. The anterior margin heights of vertebral body in the MIPPSO group and TOPSO group were $90.1 \pm 3.0 \%$ and $89.5 \pm 3.75 \%$ on the post-operative 7 th day, and $88.2 \pm 2.3 \%$ and $87.6 \pm 2.9 \%$ in the post-operative 1st year, respectively. The anterior margin heights of the vertebral body of patients in both groups on the 7 th day and in the 1st year post-operation were all significantly increased when compared to pre-operation $(\mathrm{P}<0.05)$, but there

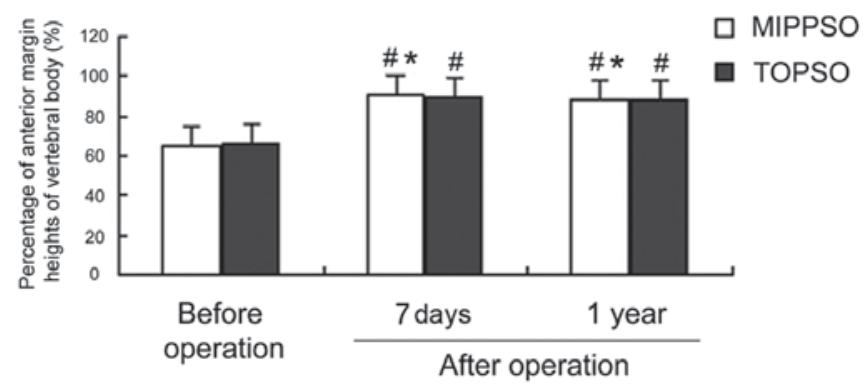

Figure 5. Comparisons of anterior margin heights of vertebral body (imaging index) of two groups of patients. Compared to the pre-operation, ${ }^{\#} \mathrm{P}<0.05$; compared to the TOPSO group, ${ }^{*} \mathrm{P}>0.05$.

were no statistically significant differences between the groups $(\mathrm{P}>0.05)$ (Fig. 5).

Post-operative complications. There was no orientation error, osteosynthesis failure, cerebrospinal fluid leakage and iatrogenic nerve injury in either group after operation. Seventy patients were treated and discharged.

\section{Discussion}

The thoracolumbar vertebra fracture is often caused by highenergy violent injuries, which are closely correlated with the anatomy of the spine. T10-L2, which is a relatively small area of thoracolumbar segment, is the region where thoracic kyphosis gradually turns into the lumbar lordosis. Due to a lack of protection of costovertebral structure, stress cannot be transmitted to a larger sagittal articular surface, which makes it easier to cause a spinal injury (8). Surgery is the best therapy in the treatment of spinal injury $(9,10)$. Currently, there are three pathways of surgery which include the anterior approach, posterior approach and anterior and posterior combined approach, of which the most commonly used mode is the posterior pedicle screw internal fixation. However, the open reduction and internal fixation has a large trauma, in which loose nails and broken nails as well as post-operative complications such as a loss of correction can easily occur (11). With the enhancement in the quality of life and aesthetic requirements, minimally invasive percutaneous pedicle screws osteosynthesis has gradually highlighted its superiority in clinical practice.

With strong axial stress and significant effect of distraction, the pedicle screw system can make the anterior and posterior ligament fully extended, which is conducive to the recovery of the height of injured vertebral body in order to produce the effect of clamping plate between the ligament and soft tissue in order to achieve indirect decompression, as well as to correct kyphosis deformity, thereby achieving reduction, fixation and early functional exercise. In the study of Palmisani et al (12), minimally invasive percutaneous pedicle screws osteosynthesis were used to treat 51 patients with a thoracolumbar vertebra fracture.

During the follow-up within 1-2 years, results showed that the recovery of patients who received osteosynthesis surgery was rapid, with fewer complications, and obtained satisfactory clinical and functional effects. The results of this study revealed 
that the pain of patients in both groups on post-operative 1st and 3rd day were relieved when compared to the pre-operation $(\mathrm{P}<0.05)$, of which the ease of pain in the MIPPSO group was more significant $(\mathrm{P}<0.05)$. The use of minimally invasive percutaneous osteosynthesis, which has a small surgical incision, can effectively reduce the muscle stripping surface of the waist and back, alleviate soft tissue injury, further avoid post-operative back pain and reduce the pain of patients, thereby improving the clinical effects. With the shortest fusion segment, the strong pedicle screw osteosynthesis system can achieve the effect of good orthopedics and maintain a threedimensional position of spine in order to further retain the motion segment (13). The differences of the Cobb's angle and anterior margin height of vertebral body of patients in both groups before the operation were not statistically significant and were therefore comparable $(\mathrm{P}>0.05)$. When comparing each group to itself between the pre-operation and post-operation, the Cobb's angles and anterior margin heights of vertebral body were significantly ameliorated $(\mathrm{P}<0.05)$. However, there were no statistically significant differences between the groups, whether pre-operation or post-operation $(\mathrm{P}>0.05)$, which are consistent with reports in literature (14). The results indicate that minimally invasive percutaneous pedicle screws osteosynthesis in the treatment of thoracolumbar vertebra fracture is beneficial in order to correct the posterior convex angle and recover the vertebral height of injured vertebra, so that the gastrointestinal, cardiac and cerebral function abnormalities, infection, spinal deformity and other complications can be reduced and the pain can be relieved effectively.

In this study, the length of surgical incision, duration of operation, bleeding volume of operation and length of hospital stay of patients in both groups were compared, and we found that the MIPPSO group had advantages of small incision, short duration of operation, less bleeding and shorter hospital stay, which shows a statistically significant difference in each parameter between the groups of data $(\mathrm{P}<0.05)$. Percutaneous pedicle screw osteosynthesis is characterized by not only a simple operation, a shorter operation time and less bleeding but also a strong fixation. In addition, it allows patients to get out of bed early, which helps avoiding multiple complications caused by a long-term bed rest, which is conducive to the early functional rehabilitation training of patients.

The body produces a special form of trauma-stress response during the process of surgery, which can cause local injury and organ dysfunction; thus, surgeons try to reduce the trauma of surgery. Currently, TNF- $\alpha$, IL- 6 and other cytokines, as well as CRP and CK, are the main indicators in the evaluation of trauma stress. Early in the 1930s, C-reactive protein (CRP) was discovered as a type of protein that can react with the pneumococcal capsular component $\mathrm{C}$ polysaccharide. The serum CRP concentra-tion is relatively low in the normal human body, however, in inflammation, burns, surgery, trauma and other stress states, the CRP concentration can be increased within $6 \mathrm{~h}$ after tissue trauma, reaching a peak in one to three days $(15,16)$, which is 2,000 times higher than the normal physiological value. As a specific stress marker, CRP is one of the most sensitive acute phase proteins produced by the stress response. The change in serum CRP concentration is positively correlated to the degree of surgical trauma, which is a relatively sensitive item that reflects the degree of tissue injury (17). None of the patients in this study showed signs of infection, which suggests that the elevated serum CRP was mainly caused by surgical trauma stress. The post-operative serum CRP levels of both groups were higher compared to the pre-operation $(\mathrm{P}<0.05)$, which was more significant in the TOPSO group $(\mathrm{P}<0.05)$. The CK content is most abundant in skeletal muscle, which is also found in the myocardium, brain and other tissues.

Generally, it is difficult for creatine kinase to pass through the cell membrane, and if it is released into the blood then there is the possibility that the cell membrane is damaged. The concentration of creatine kinase, which is low in normal human blood circulation, is released into the blood in the case that muscle cell membrane is injured and cell permeability is increased by mechanical agents such as ischemia, hypoxia and other factors. The increase in creatine kinase activity in serum is correlated with the degree of muscle stripping, the range and the time. Thus, creatine kinase is a better indicator in the assessment of muscle injury (18-24). Our results demonstrate that creatine kinase activities post-operation in both groups were significantly higher than those pre-operation $(\mathrm{P}<0.05)$. In addition, the serum creatine kinase activities were higher in the TOPSO group than the MIPPSO group at post-operative 24 and $48 \mathrm{~h}$, suggesting that minimally invasive surgery could relieve the degree of post-operative pain of waist and back via a reduction in the trauma to paravertebral muscle, which has obtained an ideal clinical effect.

In conclusion, the effect of minimally invasive percutaneous pedicle screws osteosynthesis is similar to that of the traditional open surgery. However, the technique of MIPPSO has the advantages of small trauma, less bleeding, short duration of operation, rapid post-operative recovery, light pain, less economic cost, and better aesthetic effect, and is therefore worthy of clinical promotion.

\section{References}

1. Zairi F, Aboukais R, Marinho P, Allaoui M and Assaker R: Minimally invasive percutaneous stabilization plus balloon kyphoplasty for the treatment of type A thoraco lumbar spine fractures: Minimum 4 year's follow-up. J Neurosurg Sci 58: 169-175, 2014.

2. Alizadeh M, Kadir MR, Fadhli MM, Fallahiarezoodar A Azmi B, Murali MR and Kamarul T: The use of X-shaped cross-link in posterior spinal constructs improves stability in thoracolumbar burst fracture: A finite element analysis. J Orthop Res 31: 1447-1454, 2013.

3. Kim DY, Lee SH, Chung SK and Lee HY: Comparison of multifidus muscle atrophy and trunk extension muscle strength: Percutaneous versus open pedicle screw fixation. Spine 30 : 123-129, 2005.

4. Magerl F: External skeletal fixation of the lower thoracic and the lumbar spine. In: Current Concepts of External Fixation of Fractuers. Unthoff HK and Stahl E (eds). Spring Verlag, New York, NY, pp353-366, 1982.

5. Grossbach AJ, Dahdaleh NS, Abel TJ, Woods GD, Dlouhy BJ and Hitchon PW: Flexion-distraction injuries of the thoracolumbar spine: Open fusion versus percutaneous pedicle screw fixation. Neurosurg Focus 35: E2, 2013.

6. Huskisson EC: Measurement of pain. Lancet 2: 1127-1131, 1974.

7. Huang QS, Chi YL, Wang XY, Mao FM, Lin Y, Ni WF and Xu HZ: Comparative percutaneous with open pedicle screw fixation in the treatment of thoracolumbar burst fractures without neurological deficit. Zhonghua Wai Ke Za Zhi 46: 112-114, 2008 (In Chinese).

8. Smith HE, Anderson DG, Vaccaro AR, Albert TJ, Hilibrand AS, Harrop JS and Ratliff JK: Anatomy, biomechanics, and classification of thoracolumbar injuries. Semin Spine Surg 22: 2-7, 2010. 
9. Gu Y, Zhang F, Jiang X, Jia L and McGuire R: Minimally invasive pedicle screw fixation combined with percutaneous vertebroplasty in the surgical treatment of thoracolumbar osteoporosis fracture. J Neurosurg Spine 18: 634-640, 2013.

10. Gu YT, Zhu DH, Liu HF, Zhang F and McGuire R: Minimally invasive pedicle screw fixation combined with percutaneous vertebroplasty for preventing secondary fracture after vertebroplasty. J Orthop Surg 10: 31, 2015.

11. Park HY, Lee SH, Park SJ, Kim ES, Lee CS and Eoh W: Minimally invasive option using percutaneous pedicle screw for instability of metastasis involving thoracolumbar and lumbar spine : A case series in a single center. J Korean Neurosurg Soc 57: 100-107, 2015

12. Palmisani M, Gasbarrini A, Brodano GB, De Iure F, Cappuccio M, Boriani L, Amendola L and Boriani S: Minimally invasive percutaneous fixation in the treatment of thoracic and lumbar spine fractures. Eur Spine J 18 (Suppl 1): 71-74, 2009.

13. Gaines RW Jr: The use of pedicle-screw internal fixation for the operative treatment of spinal disorders. J Bone Joint Surg Am 82: 1458-1476, 2000.

14. Ni WF, Huang YX, Chi YL, Xu HZ, Lin Y, Wang XY, Huang QS and Mao FM: Percutaneous pedicle screw fixation for neurologic intact thoracolumbar burst fractures. J Spinal Disord Tech 23: $530-537,2010$

15. Hildebrand F, Pape HC and Krettek C: The importance of cytokines in the posttraumatic inflammatory reaction. Unfallchirurg 108: 793-794, 796-803, 2005 (In German).

16. Laporta Baez Y,Palomero Rodriguez MA, De Vicente SánchezJC, Sanabria Carretero P, Al Kassam Martínez D, Pérez Ferrer A, Muriel Villoria C and Gilsanz Rodríguez F: C-reactive protein in the diagnosis of postoperative infection in pediatric patients: A prospective observational study of 103 patients. J Pediatr Surg 46: 1726-1731, 2011.

17. Kragsbjerg P, Holmberg H and Vikerfors T: Serum concentrations of interleukin-6, tumour necrosis factor-alpha, and $\mathrm{C}$-reactive protein in patients undergoing major operations. Eur J Surg 161: 17-22, 1995
18. Pokora I, Kempa K, Chrapusta SJ and Langfort J: Effects of downhill and uphill exercises of equivalent submaximal intensities on selected blood cytokine levels and blood creatine kinase activity. Biol Sport 31: 173-178, 2014.

19. Bagai A, Schulte PJ, Granger CB, Mahaffey KW, Christenson RH, Bell G, Lopes RD, Green CL, Lincoff AM, Armstrong PW, et al: Prognostic implications of creatine kinase-MB measurements in ST-segment elevation myocardial infarction patients treated with primary percutaneous coronary intervention. Am Heart J 168: 503-511, 2014.

20. Kim S, Um TH, Cho CR and Jeon JS: False-positive elevation of creatine kinase MB mass concentrations caused by macromolecules in a patient who underwent nephrectomy for renal cell carcinoma. Ann Lab Med 34: 405-407, 2014.

21. Nnadi E, Manafa P, Okocha E, Chukwuma G and Aneke J: Evaluation of creatine kinase activity and inorganic phosphate concentration in adult nigerian homozygous and heterozygous hemoglobin phenotypes. Ann Med Health Sci Res 4: 697-700, 2014.

22. Johnsen SH, Lilleng H and Bekkelund SI: Creatine kinase as predictor of blood pressure and hypertension. Is it all about body mass index? A follow-up study of 250 patients. J Clin Hypertens (Greenwich) 16: 820-826, 2014.

23. Laoutidis ZG and Kioulos KT: Antipsychotic-induced elevation of creatine kinase: A systematic review of the literature and recommendations for the clinical practice. Psychopharmacology (Berl) 231: 4255-4270, 2014.

24. Behringer M, Montag J, Kilian Y, Mccourt M and Mester J: The repeated bout effect: Is the blunted creatine kinase response an effect of an altered enzyme inactivation kinetic? J Sports Med Phys Fitness 55: 1431-1437, 2015. 